\title{
WHEEL/RAIL TANGENTIAL CONTACT STRESS EVALUATION BY MEANS OF THE MODIFIED STRIP METHOD
}

The article deals with the way of calculating tangential stresses over non-elliptical contact patch where Kalker's simplified method FASTSIM can be used advantageously. This method named FASTSTRIP is adapted for non-elliptical contact area calculated by means of the Strip method. This method is almost as quick as FASTSIM and the results are similar to the CONTACT results. This method may be useful for rail vehicles in track dynamics computation.

Keywords: FASTSIM, FASTSTRIP, modified Strip method, wheel/rail contact, contact tangential stress evaluation, optimized computation procedure.

\section{Introduction}

The rail / wheel contact relations for purposes of rail vehicles dynamics are often calculated by means of Hertz method [1] and Kalker's simplified method applied in the program code FASTSIM [2]. Kalker's variation method [3] used to be considered as an etalon for contact patch and contact stress between railway wheel and rail calculation. Normal stresses and contact patches areas are assessed with the program code NORM [3], tangential stresses and tangential forces with the program code TANG [3]. The computation with Kalker's variation method takes longer time than the computation with the simplified method. This is the reason why the variation method is not widely used for rail vehicles dynamics computation and the simplified method is preferred for this purpose. The results gained with the simplified method are partially different (but acceptable) from the results gained with the variation method. The most significant difference consists in the contact area shape and size calculated in program FASTSIM that always presupposes to be elliptical. The Strip method procedures [4] give more opportunities to solve the contact with respect to non-elliptical contact patch. Our aim is to create the calculation procedure of "FASTSIM" type - we can name it "FASTSTRIP" for calculation of stresses over a non-elliptical contact area. We derived the procedures for fast non-elliptical contact patch calculation [5] as a presupposition of tangential forces computation. The resultant values of our brand new procedure presented in this article are closer to calculation results achieved by Kalker's variation method [3], while the computation speed is similar to the computation speed of FASTSIM [2]. The authors' initial remarks on the wheel/rail tangential contact stress evaluation by means of the modified Strip method can be found in [6] and first article in [7].

\section{Prerequisites}

To begin with, we calculate the moduli of shear elasticity for wheel and rail materials [3]:

$$
\begin{aligned}
G_{1} & =\frac{E_{1}}{2 \cdot\left(1+v_{1}\right)}, G_{2}=\frac{E_{2}}{2 \cdot\left(1+v_{1}\right)}, \\
G_{12} & =\frac{2}{\frac{1}{G_{1}}+\frac{1}{G_{2}}}
\end{aligned}
$$

where:

$G_{1}, G_{2}$ - moduli of shear elasticity,

$E_{1}, E_{2}$ - moduli of elasticity,

$v_{1}, v_{2}$ - Poisson's ratios,

The contact area assembled from strips and normal stress above the strips is calculated with the Strip method.

The output parameters from the Strip method that come into the modified procedure are:

$N$ - number of strips,

$y_{i}$ - centre of i-th strip coordinate,.

$y_{d}$ - half-length of the strips,

$x_{d i}$ - half-length of the i-th strip,

$p_{o i}$ - normal stress in the middle of i-th strip,

$A_{N S}$ - area of all strips.

\footnotetext{
* Tomas Lack, Juraj Gerlici

Department of Transport and Handling Machines, Faculty of Mechanical Engineering, University of Zilina, Slovakia

E-mail: tomas.lack@fstroj.uniza.sk
} 
We used the modified "FASTSTRIP" method for the tangential stresses computation. Figure 1 shows the program dialog with graphical output of results.

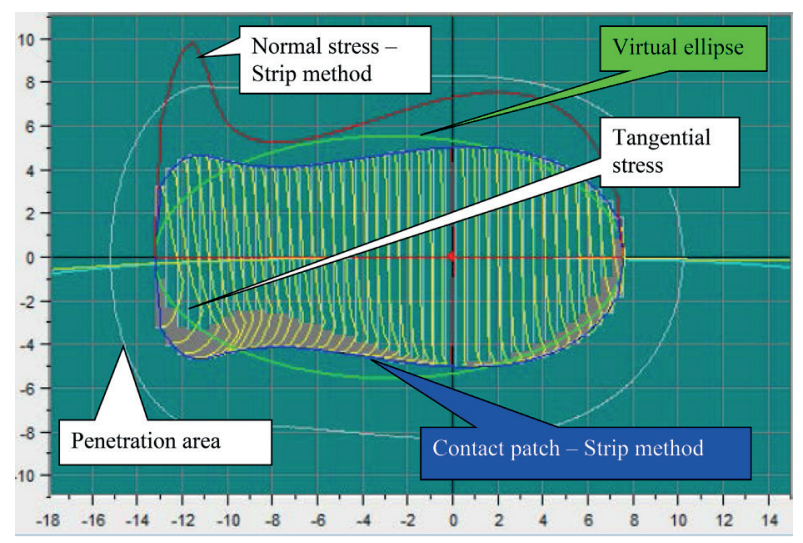

Fig. 1 Plot of AreaNORM and AreaFASTSTRIP

against wheelset treads profiles lateral movement

We use this method for computation of the stresses in the non-elliptic contact patch area. Before the computation, it is needed to compute or find out the virtual ellipse parameters:

Slips $S_{x}, S_{y}$ and spin $\psi$ are calculated from the geometrical relations.

For constants $C_{11}, C_{22}, C_{23}$ determination, the imaginary elliptical contact patch with the $b$ semi-axis:

$b=\frac{y_{N}-y_{1}}{2}+y_{d}$

the $a$ semi-axis:

$a=\frac{A_{N S}}{\pi \cdot b}$

and with the ellipse centre coordinate $y_{0}$ :

$y_{0}=\frac{y_{1}+y_{N}}{2}$

will be used.

For the semi axes proportion the following relation is valid:

$D=\frac{b}{a}$

We will set $C_{11}, C_{22}, C_{23}$ constants for the given $D$ parameter and $\mu$ friction coefficient.

For $C_{1}, C_{2}, C_{3}$ constants the following relations are valid:

$C_{1}=\frac{9}{32} \cdot C_{11}, C 2=\frac{9}{32} \cdot C_{22}, C_{3}=\frac{3 \sqrt{D}}{\pi} \cdot C_{23}$
We determine the number of splitting up the strips in the longitudinal direction:

$n_{x}=\operatorname{Int}(8 \cdot a)$

The tangential forces $T_{x}, T_{y}$ and the spin moment $M_{z}$ are set to be zero at the beginning.

\section{Mathematical computational alghoritm}

The mathematical computational algorithm is schematically depicted in the flow chart in Fig. 2.

\subsection{Calculation at the i-th strip (A):}

For the i-th strip position the coordinate in the imaginary ellipse area is as follows:

$y_{e i}=\frac{y_{i}}{b}$

and for its width the following holds:

$y_{e d}=\frac{2 \cdot y_{d}}{b}$.

For tangential maximum stress the relation mentioned below holds:

$T_{0}=\mu \cdot p_{0 i}$

where:

$\mu$ is the friction coefficient.

We will calculate the following constants:

$C=\frac{G_{12}}{T_{0}}, U_{x}=C \cdot C_{1} \cdot s_{x}, U_{y}=C \cdot C_{2} \cdot s_{y}$,

$F_{x}=C \cdot C_{3} \cdot b \cdot \psi, F_{y}=C \cdot C_{3} \cdot a \cdot \psi$

For the half-length of the strip the following relation holds:

$a_{e i}=\frac{x_{d i}}{a}$.

For a calculating step we have:

$\delta_{x}=\frac{a_{e i}}{n_{x}}$

and for the area element:

$A_{e}=y_{e d} \cdot \delta_{x}$

For the strip slip in the $x$-axis direction we have:

$s_{x i}=U_{x}-F_{x} \cdot y_{e i}$. 


\subsection{Calculation over the length of i-th strip (B):}

The $p_{x}, p_{y}$ tangential stresses are set to be zero at the beginning of the calculation over the strip length.

The $x_{e}$ coordinate is being changed in interval $\left\langle a_{e i}-\frac{\delta_{x}}{2},-a_{e i}+\frac{\delta_{x}}{2}\right\rangle$ with a step of $\delta_{x}$.

For the current slip in the $y$ direction following holds:

$s_{y i}=U_{y}+F_{y} \cdot \frac{\left(a_{e i}+x_{e}\right)}{2}$.

For tangential stresses over the area element with coordinates $\left(x_{e}, y_{e i}\right)$ following relation holds:

$p_{x}=p_{x}-s_{x i} \cdot\left(a_{e i}-x_{e}\right), p_{y}=p_{y}-s_{y i} \cdot\left(a_{e i}-x_{e}\right)$

Then the stress amplitude and maximum feasible amplitude ratio will be calculated

$p=\frac{\sqrt{p_{x}^{2}+p_{y}^{2}}}{a_{e i}^{2}-x_{e}^{2}}$.

If $p>1$, then:

$p_{x}=\frac{p_{x}}{p}, p_{y}=\frac{p_{y}}{p}$.

We will calculate the $T_{e x}, T_{e y}$ tangential forces and $M_{e z}$ spin moment for an area element.

$T_{e x}=p_{x} \cdot A_{e}, T_{e y}=p_{y} \cdot A_{e}$,

$m_{e z}=\left(p_{x} \cdot y_{e i}-p_{y} \cdot x_{e}\right) \cdot A_{e}$

These forces and spin moment are added to $T_{x}, T_{y}$ forces and $M_{z}$ spin moment:

$T_{x}=T_{x}+T_{e x}, T_{y}=T_{y}+T_{e y}, M_{z}=M_{z}+M_{e z}$

Having completed the calculation over all the strips, the $T_{x}, T_{y}$ forces and the $M_{z}$ spin moment will be divided by the $T_{z}=\frac{\pi}{2}$ constant.

$T_{x}=\frac{T_{x}}{T_{z}}, T_{y}=\frac{T_{y}}{T_{z}}, M_{z}=\frac{M_{z}}{T_{z}}$.

To obtain true values of $T_{x}, T_{y}$ and $M_{z}$ spin moment, the values calculated from (22) have to be multiplied by the constant $T$ :

$T=N \cdot \mu$

$T_{x}=T_{x} \cdot N \cdot \mu, T_{y}=T_{y} \cdot N \cdot \mu, M_{z}=M_{z} \cdot N \cdot \mu$

where:

$\mu$ is a friction coefficient,

$N$ is a normal force.

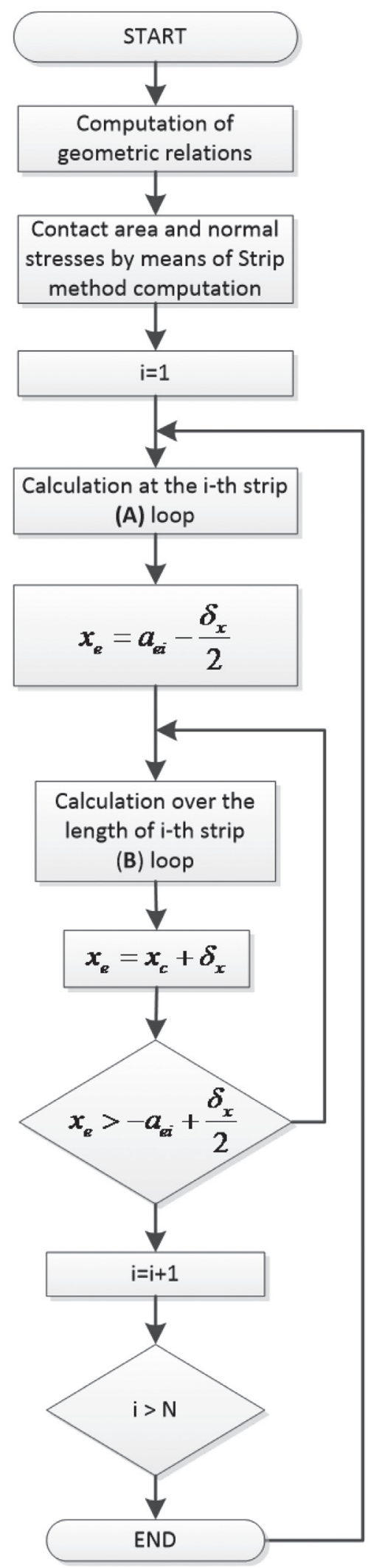

Fig. 2 Flow chart of the procedure 


\section{Results and validation}

We analyzed the contact patch area, contact stress between the wheel equipped by S1002 tread profile and UIC60 rail head profile inclined by 1:40. The lateral shift is in interval of (cca $-5 \mathrm{~mm}$ to $5 \mathrm{~mm}$ ).

\subsection{Input parameters}

The wheel force is $Q=100.000 \mathrm{~N}$. We used our fast strip method [5] for the computation of contact patches and contact stresses and we compared our results with the results obtained by Kalker's Contact-NORM method [3].

In Table 1 are summarized input parameters: yw [mm] is lateral shift of wheels profiles over the rail heads profiles, Fn [N] is a normal force, $\tan (\mathrm{Gama})$ is the value of Tangent Gamma function that is the contact area angle tangent in the contact point. Gama [rad] is the same angle expressed in radians. Sx and Sy are the slips (creepages in $\mathrm{x}$ and $\mathrm{y}$ directions) and Phi is expressed in $[\mathrm{rad} / \mathrm{mm}]$. The difference with the FASTSIM method is that the computation is executed along the strip separately, regardless of the strip size - whether it is smaller or longer than the virtual ellipse border.

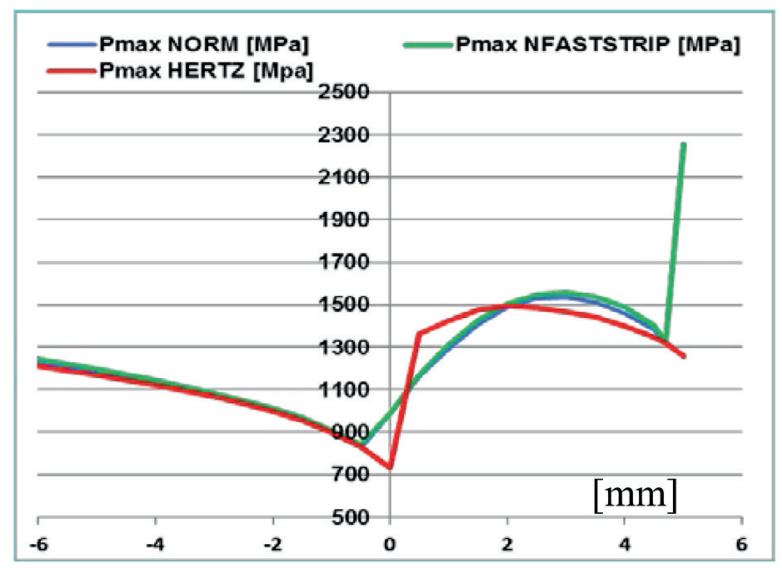

Fig. 3 Plot of Pmax CONTACT-NORM, Pmax NFASTSTRIP and Pmax HERTZ against wheelset treads profiles lateral movement

\begin{tabular}{|c|c|c|c|c|c|c|}
\hline yw & Fn [N] & $\tan ($ Gama $)$ & Gama [rad] & Sx [-] & Sy [-] & Phi [rad/mm] \\
\hline-5 & 100004 & -0.008948 & -0.00894776 & 0.001645 & 0 & 0.000019 \\
\hline-4 & 100004.7 & -0.009655 & -0.00965470 & 0.001295 & 0 & 0.000021 \\
\hline-3 & 100006.0 & -0.010960 & -0.01095956 & 0.001054 & 0 & 0.000024 \\
\hline-2.5 & 100007.1 & -0.011927 & -0.01192643 & 0.000953 & 0 & 0.000026 \\
\hline-2 & 100008.6 & -0.013144 & -0.01314324 & 0.000859 & 0 & 0.000029 \\
\hline-1.5 & 100010.9 & -0.014776 & -0.01477492 & 0.000773 & 0 & 0.000032 \\
\hline-1 & 100014.2 & -0.016839 & -0.01683741 & 0.000684 & 0 & 0.000037 \\
\hline-0.5 & 100019.5 & -0.019748 & -0.01974543 & 0.000595 & 0 & 0.000043 \\
\hline 0 & 100029.0 & -0.024072 & -0.02406735 & 0.000000 & 0 & 0.000052 \\
\hline 0.5 & 100222.8 & -0.066581 & -0.06648288 & -0.000595 & 0 & 0.000145 \\
\hline 1 & 100249.4 & -0.070613 & -0.07049599 & -0.000684 & 0 & 0.000153 \\
\hline 1.5 & 100282.8 & -0.075148 & -0.07500702 & -0.000773 & 0 & 0.000163 \\
\hline 2 & 100317.3 & -0.079742 & -0.07957362 & -0.000859 & 0 & 0.000173 \\
\hline 2.5 & 100360.8 & -0.084998 & -0.08479419 & -0.000953 & 0 & 0.000184 \\
\hline 3 & 100411.8 & -0.090812 & -0.09056359 & -0.001054 & 0 & 0.000197 \\
\hline 3.5 & 100472.2 & -0.097318 & -0.09701251 & -0.001166 & 0 & 0.000210 \\
\hline 4 & 100550.2 & -0.105034 & -0.10465029 & -0.001295 & 0 & 0.000227 \\
\hline 4.5 & 100652.9 & -0.114436 & -0.11394035 & -0.001449 & 0 & 0.000247 \\
\hline 4.7 & 100703.2 & -0.118818 & -0.11826354 & -0.001519 & 0 & 0.000256 \\
\hline 5 & 100799.7 & -0.126724 & -0.12605211 & -0.001645 & 0 & 0.000273 \\
\hline
\end{tabular}




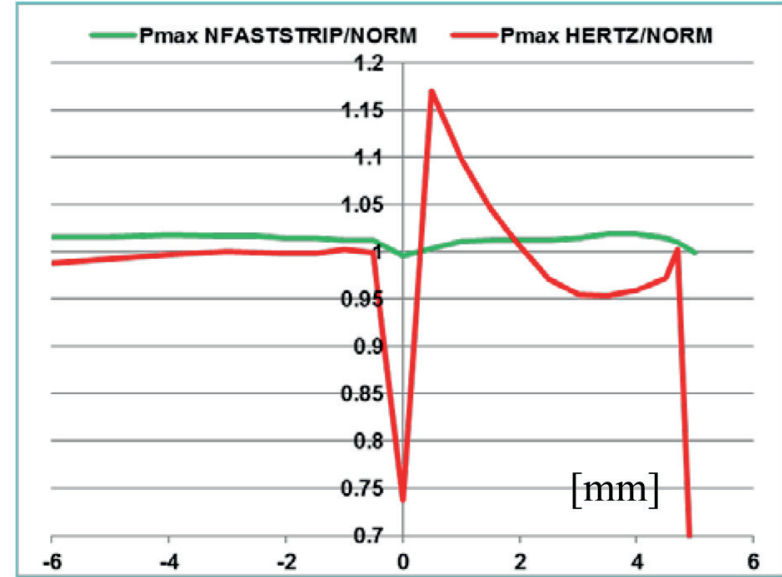

Fig. 4 Plot of Pmax NFASTRSTRIP/NORM and Pmax HERTZ/ NORM [-] proportional comparison of evaluated quantities

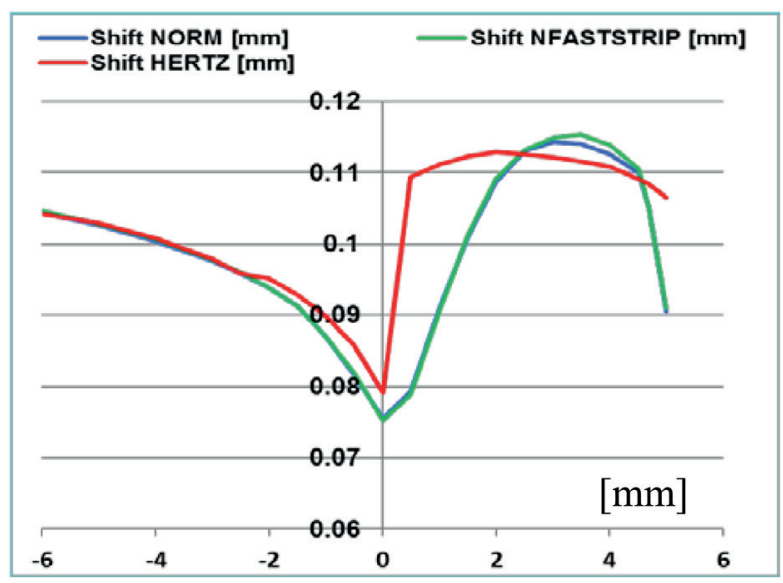

Fig. 5 Plot of Shift NORM, Shift FASTSTRIP and Shift HERTZ against wheelset treads profiles lateral movement

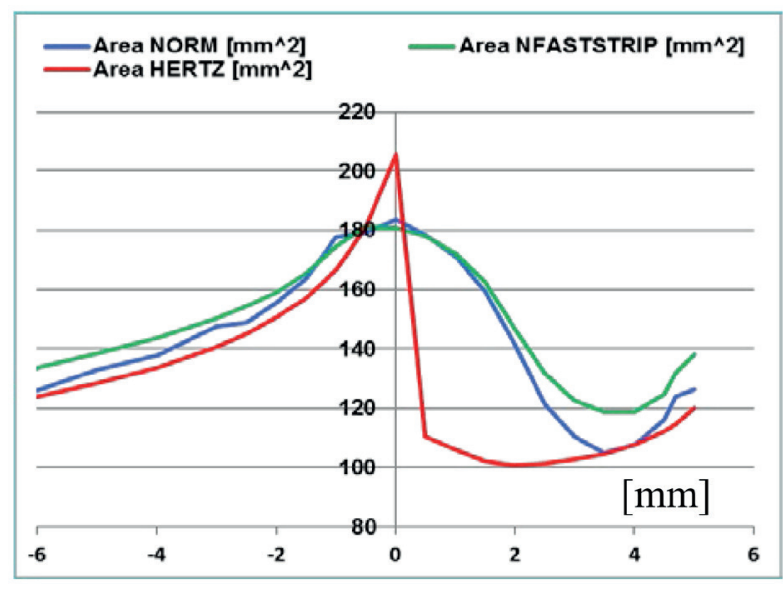

Fig. 6 Plot of Area NORM, Area FASTSTRIP and Area HERTZ against wheelset treads profiles lateral movement

\subsection{Results of tangential stresses calculation}

Tangential stresses, $T_{y}, T_{y}$ forces and $M_{z}$ moment for separate strips are computed by means of the $T_{x}, T_{y}$ and $M_{z}$ computed by "CONTACT-TANG", "FASTSIM" and "FASTSTRIP“ methods for the purpose of comparison.

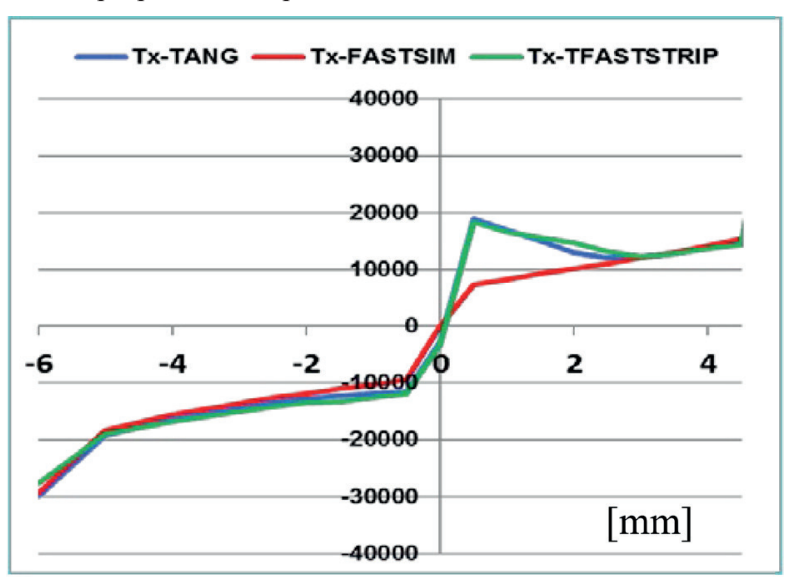

Fig. 7 Plot of $T_{x}[N]$ against wheelset treads

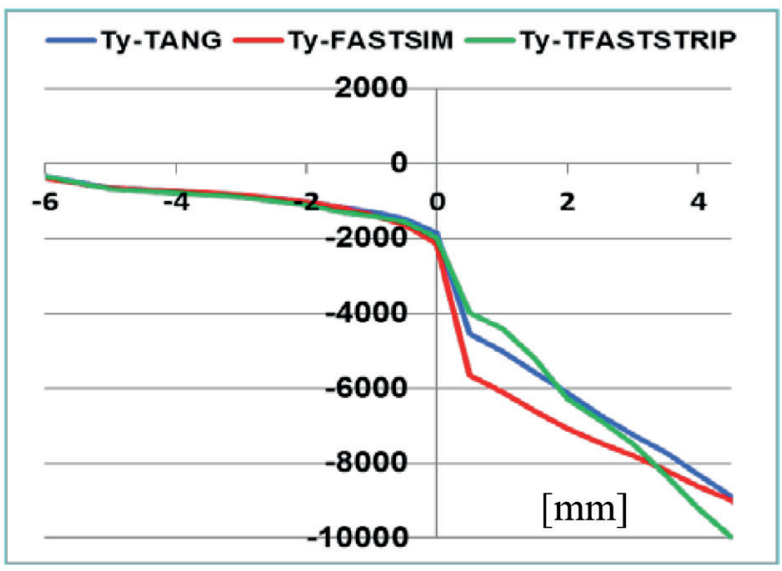

Fig. 8 Plot of $T_{\nu}[N]$ against wheelset treads profiles lateral movement

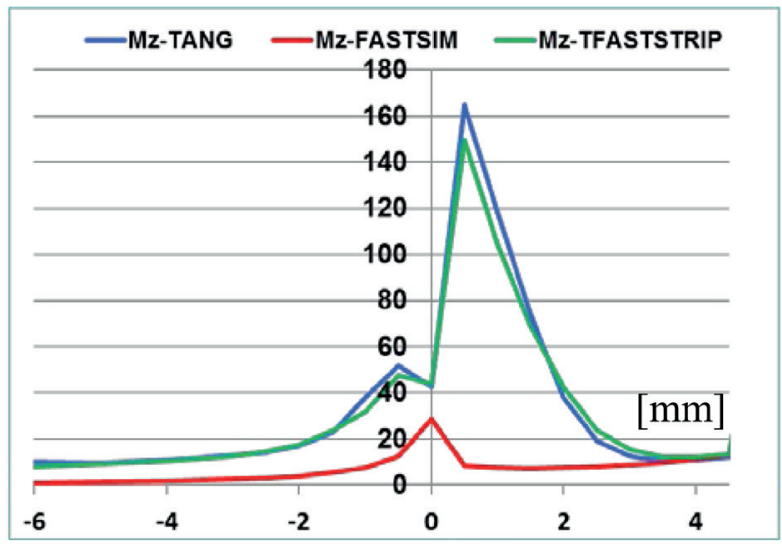

Fig. 9 Plot of $M_{z}[N . m]$ against wheelset treads 


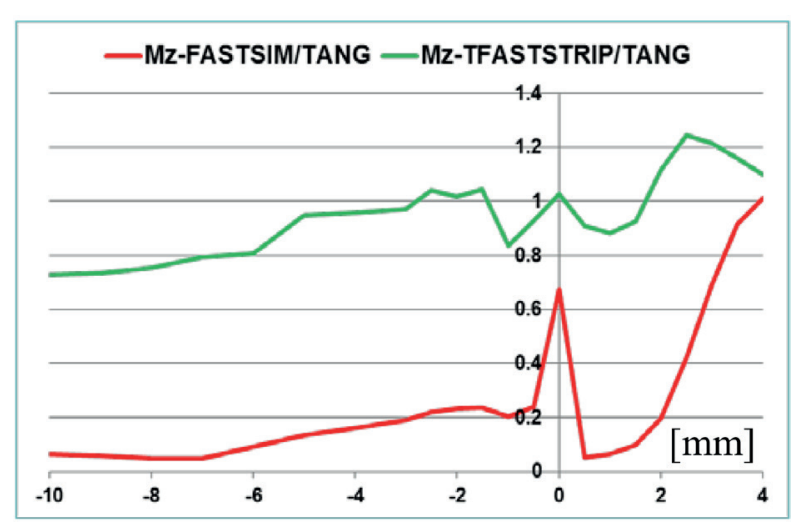

Fig. 10 Plot of $M_{z}[N . m]$ - Proportional comparison of evaluated quantity

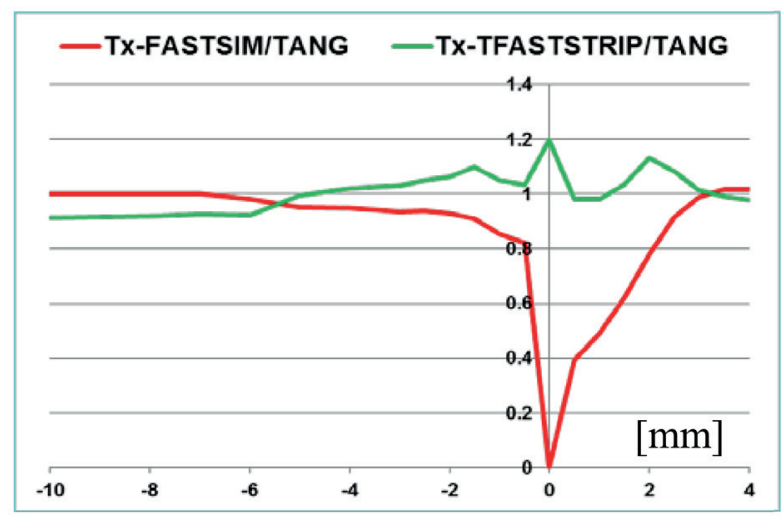

Fig. 11 Plot of $T_{x}[$ N]- Proportional comparison

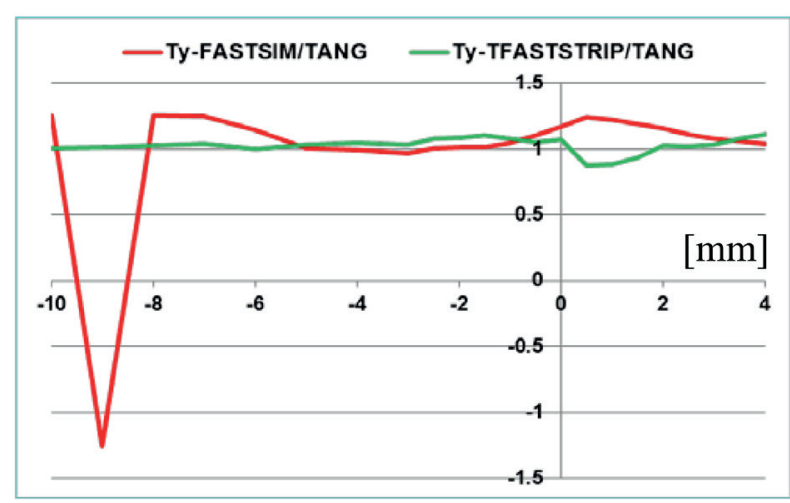

Fig. 12 Plot of Ty [N]- Proportional comparison of evaluated quantity

\section{Conclusions}

Our aim is to create the calculation procedure of "FASTSIM" type. We named this procedure for calculation of stresses over non-elliptical contact area "FASTSTRIP“. The result values are closer to the results achieved by Kalker's variation method results and the computation speed is similar to the computation speed of FASTSIM. This method is adapted for a non-elliptical contact area calculated by means of the Strip method [5]. This method utilizes the FASTSIM theory [2] as a calculation engine for tangential stress assessment. The calculation procedure is outlined in Fig. 2 in which a flowchart with two program loops is illustrated. These loops are in detail described in the part "Mathematical model". Results and validation follow. In Table 1 are some input parameters, Figs. 3, 4, 5 and 6 compare the results gained by means of NORM [3] and our calculation procedure [5] shift, area and pmax. They express the reality that the ground input parameters for tangential forces calculations are mutually very close.

Figures 7, 8, 9 and 10 give results of tangential stresses calculation for input parameters. The curves of dependencies (Tx, Ty Mz) calculated with TANG [3] and FASTSTRIP are shown in graphs. For better resolution are these curves shown in Figs. 11 and 12 as comparative proportional curves. The meaning or importance of the procedure FASTSTRIP for us or anybody who writes his/her own code is in the fact that this procedure can be implemented into the code for computation of rail vehicles dynamics offering the advantage of fast computations. Of course, the field may be analysed from other points of view [8, 9 and 10] where multi-software platforms are presented. The field of wheel /rail contact task is very close to the computational analysis of contact stress distribution in the case of mutual slewing of roller bearing rings [11 and 12], or from the more sophisticated field [13, 14 and 15].

\section{Acknowledgement}

The work was supported by the Scientific Grant Agency of the Ministry of Education of the Slovak Republic and the Slovak Academy of Sciences in project No. 1/1098/11: "Stress Distribution in a Braked Railway Wheel". No. 1/0347/12: "Railway wheel tread profile wear research under the rail vehicle in operation conditions simulation on the test bench", project No. 1/0383/12: "The rail vehicle running properties research with the help of a computer simulation." and the project No. APVV-0842-11: "Equivalent railway operation load simulator on the roller rig".

Research-Educational Center of Rail Vehicles (VVCKV) 


\section{References}

[1] JOHNSON, K. L.: Contact Mechanics. Cambridge university press Cambridge, p. 510. 1987.

[2] KALKER, J. J. A Fast Algorithm for Simplified Theory of Rolling Contact. Vehicle systems dynamics, 11, 1982, 1-13.

[3] KALKER, J. J.: Three-dimensional Elastic Bodies in Rolling Contact. Kluwer academic publishers: Dordrecht, 1990.

[4] KNOTHE, K., HUNG, L.-T.: Ermittlung der Normalspannungs-verteilung beim Kontakt von Rad und Schiene. Forsch. Ing.-Wes. 49, 1983, 79-83, 1983.

[5] LACK, T., GERLICI, J.: Modified Strip Method Utilisation for Wheel / rail Contact Stress Evaluation. $9^{\text {th }}$ intern. conference on contact mechanics and wear of rail/ wheel systems (CM2012) : Southwest Jiaotong University, 2012. Presented in Chengdu, 2012.

[6] LACK, T., GERLICI, J.: Wheel/rail Contact Stress Evaluation by Means of the Modified Strip Method. Communications - Scientific Letters of the University of Zilina, vol. 15, No. 3, 2013, 126-132. ISSN 1335-4205.

[7] LACK, T., GERLICI, J.: Tangential Stresses for Non-eliptical Contact Patch Computation by Means of Modified FASTIM Method. Intern. Association of Vehicles Systems Dynamics (IAVSD) $2013: 23^{\text {rd }}$ intern. symposium on dynamics of vehicles on roads and tracks, August 2013, Qingdao: Chengdu: Southwest Jiaotong University, 2013. USB key, [6] p.

[8] SAPIETOVA, A., SAGA, M., NOVAK, P.: Multi-software Platform for Solving of Multibody Systems Synthesis, Communications Scientific Letters of the University of Zilina, vol. 14, No. 3, 2012, 43-48. ISSN 1335-4205.

[9] LEITNER, B.: The software tool for mechanical structures dynamic systems identification. Transport means 2011. Proceedings of the 15th international conference : October 20-21, 2011, Kaunas University of Technology, Lithuania. - ISSN 1822-296X. - Kaunas: Kaunas University of Technology, 2011. - S. 38-41.

[10] BEDNAR, R. SAGA, M., VASKO, M.: Effectivity Analysis of Chosen Numerical Methods for Solution of Mechanical Systems with Uncertain Parameters. Communications - Scientific Letters of the University of Zilina, vol. 13, No. 4, 2011, 40-45. ISSN 1335-4205.

[11] JAKUBOVICOVA, L., SAGA, M.: Computational Analysis of Contact Stress Distribution in the Case of Mutual Slewing of Roller Bearing Rings. Applied Mechanics and Materials, 2014.

[12] JAKUBOVICOVA, L., SAGA, M., VASKO, M.: Impact Analysis of Mutual Rotation of Roller Bearing Rings on the Process of Contact Stresses in Rolling Elements. Manufacturing Technology, 2013.

[13] ZMINDAK, M., RIECKY, D., SOUKUP, J.: Failure of Composites with Short Fibres. Communications - Scientific Letters of the University of Zilina, vol. 12, No. 4, 2010, 33-39. ISSN 13354205.

[14] ZMINDAK, M., NOVAK, P.: Particle Interactions in Composites Reinforced by Fibre and Spherical Inclusions. Communications Scientific Letters of the University of Zilina, vol. 11, No. 2, 2009, 13-18, ISSN 1335-4205.

[15] LEITNER, B.: Optimization of Dynamic Strength Dimension of Machine Frame by MATLAB. Transport Means - Proc. of the Intern. Conference 2012, Pages 104-107, 16 ${ }^{\text {th }}$ Intern. Conference Transport Means 2012; Kaunas; October 2012. 